\title{
Comissurotomia mitral: como evoluem os pacientes a longo prazo?
}

Antoninho S. ARNONI*, W. J. COUTO*, Jarbas J. DINKHUYSEN*, Paulo CHACCUR*, Zilda M. MENEGHELO*, Luiz Carlos Bento de SOUZA*, Adib D. JATENE**, Paulo P. PAULISTA*

ARNONI, A. S.; COUTO, W. J.; DINKHUYSEN, J. J.; CHACCUR, P.; MENEGHELO, Z. M.; SOUZA, L. C. B.; JATENE, A. D.; PAULISTA P. P. - Comissurotomia mitral: como evoluem os pacientes a longo prazo? Rev. Bras. Cir. Cardiovasc., 5(2): 106-112, 1990.

RESUMO: Procuranco saber qual a evolução a longo prazo daqueles pacientes submetidos a comissurotomia mitral há mais de 14 anos, analisamos 100 pacientes, operados entre 1962 e 1975 e que tiveram seu acompanhamento no Instituto Dante Pazzanese de Cardiologia. Muitos pacientes foram operados nesse intervalo, mas não tiveram seu seguimento em nossa Instituição e não foram considerados. Setenta e seis eram do sexo feminino e 24 do masculino, com idades variando de 11 a 50 anos, com média de 30,8 anos. Sessenta e cinco pacientes foram reoperados, sendo que em 18 ocasiōes realizou-se outra comissurotomia, em um fez-se a revascularização, em um substituição da valva tricúspide; em 45 vezes a valva foi substituída ( 43 próteses biológicas e duas metálicas). 0 tempo médio entre a primeira e a segunda cirurgias foi de 13,6 anos. Não houve óbitos na reoperação. Trinta e cinco pacientes continuam em evoluçāo da primeirs cirurgia, com um tempo médio de 17,2 anos, com um mínimo de 14 anos e um máximo de 27 anos. Dez estão no grupo funcional I, 17 no II, sete no III e um no grupo IV. Desta forma, dos 100 pacientes iniciais, 52 ainda estão com suas valvas naturais, mostrando que, apesar de a evolução da doença poder levar a alterações nas valvas operadas, a comissurotomia mitral consegue uma boa evolução a longo prazo.

DESCRITORES:comissurotomia mitral, evolução tardia; valva mitral, cirurgia.

\section{INTRODUÇÃO}

A estenose da valva mitral foi uma das primeiras lesōes a ser abordada cirurgicamente. No início, através da comissurotomia digital ou instrumental ${ }^{4,8,5}$, muitos casos foram operados satisfatoriamente. $\mathrm{O}$ advento da circulaçāo extracorpórea possibilitou a visão direta das valvas e a abertura da estenose pôde ser mais completa, com atuação não só ao nível valvar, mas também das estruturas subvalvares, separando cordas e músculos papilares $^{12,25}$. Além disso, valvas com lesōes mais severas puderam ser tratadas através da associação de comissurotomia e plastia dos maị diferentes tipos ${ }^{6,22}$. $A$ introdução dos substitutos valvares ${ }^{27}$ com o apareci- mento de próteses artificiais, biológica ou mecânica, deu chance a que o cirurgião tratasse todo tipo de lesão valvar, desde uma estenose simples até uma dupla lesão calcificada. Entretanto, as próteses artificiais não estão livres de complicações que envolvem tromboembolismo, trombose da prótese, vazamento peri-valvular, endocardite ou degeneração por calcificação.

Recentemente ${ }^{19}$, a abordagem da mitral pôde ser feita por cateter-balāo, na tentativa de dilataçāo sem necessidade de cirurgia. Inúmeros trabalhos têm sido descritos nessa área, mostrando sucesso ${ }^{16}$, complicações ${ }^{3}$ e reestenoses tardias ${ }^{25}$.

Trabalho realizado no Instituto Dante Pazzanese de Cardiologia. São Paulo, SP. Brasil.

Apresentado ao 17: Congresso Nacional de Cirurgia Cardiaca. Belo Horizonte, MG, 7 e 8 de abril, 1990.

- Do Instituto Dante Pazzanese de Cardiologia.

* Do Hospital do Coraçăo da Associação do Sanatório Sírio.

Endereço para separtas: Antoninho S. Arnoni. Av. Danta Pazzanese, 500. 04012 lbirapuera, São Paulo, SP, Brasil. 
ARNONI, A. S.; COUTO, W. J.; DINKHUYSEN, J. J.; CHACCUR, P.; MENEGHELO, Z. M.; SOUZA, L. C. B.; JATENE, A. D.; PAULISTA P. P. - Comissurotomia mitral: como evoluem os pacientes a longo prazo? Rev. Bras. Cir. Cardiovasc., 5(2): 106-112, 1990

Esses problemas têm levado todas as equipes cirúrgicas a envidar esforços no sentido de conservar o apareIho valvar do paciente e, dentro das técnicas de conservação, a comissurotomia acompanhada de liberação e abertura das estruturas subvalvares desempenha papel importante.

Por estes motivos, procuramos saber como evoluem a longo prazo os pacientes submetidos a comissurotomia mitral isolada, e quais os fatores que poderiam atuar na longevidade de uma comissurotomia. Desta forma, excluímos aqueles em que qualquer outro procedimento associado foi realizado, tais como plastias da própria mitral, plastia ou substituição da tricúspide, ou substituição de outra valva cardíaca.

\section{CASUÍSTICA E MÉTODOS}

Entre 1962 e 1975,4175 pacientes foram operados no Instituto Dante Pazzanese de Cardiologia, para correção de lesões valvares, sendo 2792 cirurgias exclusivamente sobre a valva mitral, com 1841 comissurotomias, 84 plastias e 867 substituiçōes. Nos restantes 1383 pacientes, a cirurgia foi sobre as outras valvas, ou sobre a associação de problemas mitrais com outras valvas, ou com revascularizaçāo do miocárdio.

A mortalidade nas comissurotomias mitrais foi de 1,14\%, com 21 óbitos nos 1841 pacientes.

Destes pacientes, 100 são acompanhados no IDPC desde então, com visitas periódicas à Instituição.

A finalidade do nosso trabalho foi analisar as valvas e os fatores que podem atuar sobre elas e, por esse motivo, não escolhemos os 100 pacientes consecutivos, mas sim aqueles que tiveram sua evolução em nossa Instituição.

Setenta e seis pacientes eram o sexo feminino e 24 do sexo masculino. A idade mínima foi de 11 anos e a máxima de 55 anos, com média de 30,8 anos. Setenta e dois pacientes tinham ritmo sinusal no pré-operatório e 28 tinham fibrilaçāo arterial.

A abordagem cirúrgica era, geralmente, por toracotomia ântero-lateral direita (quarto espaço intercostal), utilizando-se, excepcionalmente, a esternotomia mediana. A circulação extracorpórea era feita com a cânula arterial introduzida através da artéria femoral. A partir de 1980, temos dado preferência à esternotomia mediana e à canulaçāo da aorta ascendente.

A visāo da valva mitral foi feita com parada anóxica intemitente e normotermia, dando-se ênfase à análise do aparelho subvalvar, procedimento mantido até hoje. O teste da competência da valva foi feito tanto digitalmente como sob visão direta, após o coração recuperar bem seus batimentos. A correlação desses testes com ausculta e ecocardiograma no pós-operatório tem sido bastante satisfatório.

\section{RESULTADOS}

Os 100 pacientes analisados têm evolução média de 19,8 anos, com mínimo de 14 anos e máximo de 27 anos. Sessenta e cinco deles foram a reoperação e 35 nāo foram reoperados. Seis desses pacientes tinham sido submetidos previamente a comissurotomia digital.

Dos 65 pacientes reoperados (Tabela 1), 18 foram a nova comissurotomia, e um a revascularização do miocárdio, um a substituiçāo da valva tricúspide e 45 a substituição valvar.

O paciente revascularizado necessitou uma nova cirurgia por oclusão de ponte. Aquele em que foi substituída a valva tricúspide foi à terceira cirurgia para substituição da valva mitral (oito anos depois).

Dos 18 que foram a recomissurotomia, dois necessitaram de uma terceira cirurgia (um para nova comissurotomia e um para substituição valvar) e dois a uma quarta cirurgia (em um foram realizadas quatro comissurotomias e, no outro, três comissurotomias e uma substituição valvar).

Nas 45 substituições valvares foram empregadas duas próteses metálicas e 43 biológicas. Os dois pacientes com próteses metálicas estão em evolução, havendo suspeitas de disfunção em um deles. Dos 43 que receberam próteses biológicas, sete foram para uma terceira cirurgia com utilização de quatro próteses biológicas, um metálica, e substituição da mitral e aórtica por próteses metálicas nos dois casos restantes. Um paciente necessitou quatro cirurgias, recebendo prótese metálica na última delas.

Durante a segunda intervenção, foram realizados cinco procedimentos associados, sendo duas comissurotomias aórticas, duas plastias de tricúspide e uma prótese em posiçāo tricúspide. Duas pacientes estavam grávidas quando da reoperação e tiveram boa evolução, tanto da parte cardiaca como gestacional.

TABELA 1

PACIENTES REOPERADOS APÓS COMISSUROTOMIA MITRAL

\begin{tabular}{lr}
\hline REOPERADOS & 65 \\
Comissutoromia & 18 \\
Revascularização & 1 \\
Próteses Tricúspide & 1 \\
Próteses & 45 \\
\hline
\end{tabular}


ARNONI, A. S.; COUTO, W. J.; DINKHUYSEN, J. J.; CHACCUR, P.; MENEGHELO, Z. M.; SOUZA, L. C. B.; JATENE, A. D.; PAULISTA P. P. - Comissurotomia mitral: como evoluem os pacientes a longo prazo? Rev. Bras. Cir. CardiovasC., 5(2): $106-112,1990$.

Dos 76 pacientes do sexo feminino, $49(64,4 \%)$ foram reoperados e, dos 24 homens, $15(62,5 \%)$ foram reoperados, diferença nâo significativa estatisticamente.

Quanto ao ritmo, 72 pacientes tinham ritmo sinusal antes da primeira cirurgia e 28 tinham fibrilação atrial (FA). Trinta e três pacientes passaram de ritmo sinusal a FA na evolução pós-operatória.

Dos 72 pacientes que tinham ritmo sinusal, 43 $(59,7 \%)$ foram reoperados e dos 28 com FA, 21 (75\%) foram reoperados, diferença estatisticamente não significante.

Dos 43 reoperados que tinham ritmo sinusal prévio, $13(30,2 \%)$ foram para nova comissurotomia e 29 $(67,4 \%)$ para substituição valvar. Dos 21 com FA, cinco $(23,8 \%)$ foram para nova comissurotomia e $16(76,2 \%)$ para troca valvar. Com relação à idade na primeira cirurgia, temos que oito pacientes tinham menos de 20 anos, seis dos quais foram reoperados; 37 tinham entre 20 e 30 anos e 27 foram reoperados; 40 estavam na faixa dos 30 aos 40 , sendo que 27 foram reoperados e 15 tinham mais de 40 anos e cinco foram reoperados (Tabela 2$)$.

Trinta e cinco pacientes estão evoluindo desde a primeira cirurgia sem a necessidade de nova intervenção. A evolução vai de 14 a 27 anos, com média de 17,2 anos. Nove pacientes têm mais de 20 anos desde a primeira cirurgia, seis dos quais têm mais de 24 anos.

Dez pacientes estão atualmente no grupo funcional I da NYHA, 17 no II, sete no III e um no grupo IV. Este último tem indicação para nova cirurgia, mas recusa-se a ser operado.

Desta forma, 52 pacientes ainda estão com suas valvas naturais, mostrando que, apesar da doença poder levar a alteraçōes nas valvas operadas, a comissurotomia mitral consegue uma boa evolução a longo prazo.

\section{COMENTÁRIOS}

A cirurgia sobre valva mitral, principalmente sobre os processos estenóticos, é realizada há muitos anos, com resultados bastante satisfatórios. CUTTER \& BECK ${ }^{8}$ descreveram técnicas de comissurotomia digital,

TABELA 2

CORRELAÇĀO DAS REOPERAÇÓES COM A FAIXA ETÁRIA

\begin{tabular}{lrr}
\hline FAIXA ETARIA & & \multicolumn{1}{l}{ REOP. } \\
\hline Menos 20 anos & 8 & $6(75,0 \%)$ \\
$20-30$ anos & 37 & $27(72,9 \%)$ \\
$30-40$ anos & 40 & $27(67,5 \%)$ \\
Mais 40 anos & 15 & $5(33,3 \%)$ \\
\hline
\end{tabular}

seguidos pelos trabalhos de BAYLEN ${ }^{4}$ e HARKEN et alii $^{15}$.

A evolução tardia dos pacientes submetidos a essa técnica mostrou reestenose que necessitava nova cirurgia, principalmente porque as estruturas subvalvares não eram tratadas satisfatoriamente.

O surgimento das comissurotomias sob visão direta $^{12,23}$ possibilitou melhor análise do aparelho valvar, fazendo com que a abertura fosse mais completa e melhorasse os resultados a longo prazo.

Desde o início, a comissurotomia mitral é realizada com baixo índice de mortalidade operatória. HALSETH et alii ${ }^{14}$ mostram uma mortalidade de $1,52 \%$ em 222 pacientes operados até dezembro de 1978. HOUSMAN et alii ${ }^{18}$ referem mortalidade de $2 \%$ em sua série de pacientes e relatam que a mortalidade nas comissurotomias a "céu-fechado" varia de $4 \%$ a $7 \%$, contra $1 \%$ a $3 \%$ nas comissurotomias sob visão direta. Nos pacientes desta nossa série, operados até 1975 , a mortalidade foi de $1,14 \%$.

Apesar da baixa mortalidade e da baixa morbidade, o surgimento das valvoplastias percutâneas fez com que o número de pacientes submetidos a comissurotomia mitral diminuísse sensivelmente. Assim é que, nos dois últimos anos (janeiro de 88 a dezembro de 89), apenas 166 pacientes foram operados para realização da comissurotomia (mortalidade 0,6\%), contra 170, 198, 183, 176 e 197, respectivamente, em 1971, 1972, 1973, 1974 e 1975.

Como a reestenose é uma das dúvidas do processo de valvoplastia, procuramos saber o que aconteceu com nossos pacientes operados a longo prazo, para, no futuro, compararmos nossos resultados com os que os procedimentos invasivos das salas de hemodinâmica possam trazer.

A durabilidade dos resultados das comissurotomias mitrais está na dependência de vários fatores, tais como idade, sexo, ritmo cardíaco prévio, grupo funcional, característica da valva e presença de gradiente ou insuficiência da valva no pós-operatório imediato. HOUSMAN et alii ${ }^{18}$ consideram de importância, no prognóstico do tratamento das estenoses mitrais, o uso de medicação e a duraçāo dos sintomas. SMITH et alii ${ }^{26}$ consideram a raça como de importância prognóstica.

A maioria dos autores, entre eles AKINS et alii ${ }^{1}$ e MULLIN et alii ${ }^{24}$ consideram de importância fundamental a abertura das estruturas subvalvares para que o procedimento tenha bom resultado a curto e longo prazos. VEGA et alii ${ }^{28}$ mostram comprometimento do aparelho subvalvar em $66 \%$ de seus pacientes e referem que a separação das cordas fusionadas e a divisão dos músculos papilares levam a um prognóstico diferente. 
ARNONI, A. S.; COUTO, W. J.; DINKHUYSEN, J. J.; CHACCUR, P.; MENEGHELO, Z. M.; SOUZA, L. C. B.; JATENE, A. D.; PAULISTA P. P. - Comissurotomia mitral: como evoluem os pacientes a longo prazo? Rev. Bras. Cir. Cardiovasc., 5(2): $106-112,1990$

HIGGS et alii ${ }^{17}$ consideram a estenose residual ou a criaçāo de uma insuficiência mitral a causa mais freqüente da recorrência dos sintomas após a cirurgia. Finalmente, não podemos esquecer que o teste da valva é importante no momento da cirurgia, para que se tenha certeza da não existência de refluxo. Alguns cirurgióes testam a valva através de infusão de soro no ventrículo esquerdo, ou pelo aspirador usado no VE, ou pela passagem do cateter pela valva aórtica. Outros produzem insuficiência aórtica para poder visibilizar a mitral com o VE cheio. Nós temos testado, tanto digitalmente, como por visão direta após o coração recuperar os batimentos.

Mais recentemente, o emprego da ecocardiografia intraoperatória ${ }^{5}$ tem ajudado bastante na análise dos bons resultados imediatos. Com o aparecimento de eletrodos intra-esofágicos ${ }^{21}$, essa análise torna-se mais simples e o procedimento mais fácil de ser realizado. Ainda não empregamos a ecocardiogafia intra-operatória, de rotina em nosso Serviço, mas cremos que ela possa auxiliar na comprovação das estenoses residuais ou insuficiências.

Acreditamos, também, que o resultado a médio prazo esteja relacionado com a técnica que consiga a melhor abertura da valva sem insuficiência residual. Para que isso ocorra, há necessidade de uma ênfase à abertura das estruturas subvalvares. Essa é, ao nosso ver, a grande diferença entre as comissurotomias digitais e, mais recentemente, as valvoplastias, com. as comissurotomias sob visão direta.

GROOS et alii ${ }^{13}$ crêem que a comissurotomia leva a uma redução no fluxo turbulento de sangue pela criação de uma valva competente e bem aberta, diminuindo a fibrose progressiva e podendo evitar a necessidade de uma troca valvar futura.

Outros fatores atuam na evolução cirúrgica, e não podemos esquecer a característica da própria valva, com fibrose intensa ou calcificação. A mobilidade das cúspides prejudicadas pela calcificação pode predispor a mau resultado. HIGGS et alii ${ }^{17}$ referem que o resultado da comissurotomia depende do grau de extensão da fibrose ou da calcificação, o mesmo acontecendo com VEGA et alii ${ }^{28}$, que referem ter encontrado calcificação em $11 \%$ dos seus casos. Em nossa série, a maioria dos pacientes que tinham calcificação na valva na primeira cirurgia necessitou reoperação. Dos 35 não reoperados, três tinham cálcio e, assim mesmo, em pequena quantidade quando da primeira intervenção.

Como a maioria dos pacientes submetidos a cirurgia valvar tem suas lesōes de origem reumática, a própria evolução da doença poderia interferir nos resultados a longo prazo. CARPENTIER et alii ${ }^{6}$ revelam maior falha no tratamento nas lesōes de causa reumática. $O$ mesmo acontece com ANTUNES et alii ${ }^{2}$, quando comparam seus resultados em reumáticos com os relatados por COSGROVE et alii ${ }^{7}$ em doenças degenerativas. Segun- do GALLOWAY et alii " ${ }^{11}$, os portadores de febre reumática têm pior resultado nas cirurgias conservadoras, quando comparados a seus pacientes com doença degenerativa. DURAN et alii ${ }^{10}$ consideram que a evolução do processo reumático tem papel importante nos pacientes que necessitam reoperação.

MANTEUFFEL-SZOEGE et alii ${ }^{23}$, analisando 1700 casos de cimissurotomia, acham que a importância da febre reumática é difícil de avaliar, mas referem que, em alguns casos, pode desenvolver ou fazer progredir lesōes ou, ainda, provocar dano miocárdico. HIGGS et alii ${ }^{17}$ não responsabilizam a recorrência de febre reumática pela reestenose de seus pacientes, mas a progressão da calcificação valvar pode ter contribuído para isso em dois de seus cinco pacientes reoperados.

A idade, na época da realização da primeira cirurgia, pode ter influência. SMITH et alii ${ }^{26}$ não conseguiram encontrar significância quanto à idade como fator isolado, mas acreditam que tenha influência quando associado a outros fatores. Na nossa série, há grande diferença entre os pacientes operados antes dos 20 anos e os que foram submetidos a cirurgia com mais de 40 anos. Dos oito operados na faixa dos 20 anos, seis (75\%) foram reoperados, e dos $15 \mathrm{com}$ mais de 40 anos, apenas cinco $(33,3 \%)$ necessitaram nova intervenção, o que é estatisticamente significativo.

O sexo também tem sido considerado como um dos elementos que pode influenciar na evolução a longo prazo. $\mathrm{Na}$ análise dos nossos pacientes, não conseguimos achar diferença quanto ao sexo, já que $64,4 \%$ das mulhares e $62,5 \%$ dos homens necessitaram de reoperação na evolução a longo prazo.

O ritmo cardiaco também tem sido levado em consideração. A fibrilação atrial tende a ocorrer em processo reumático e suas seqüelas ${ }^{29}$. DEKKER et alii ${ }^{9}$ têm demonstado que a agregação de microtrombos plaquetários ocorre em valvas com doença severa e que, possivelmente, esse processo é acelerado pela fibrilação atrial. Além disso, é indiscutivel o papel da fibrilação atrial nos processos embólicos que ocorrem na estenose mitral. Alguns autores preconizam sempre o fechamento da aurícula, mesmo na ausência de trombos. A nossa conduta tem sido suturar a aurícula esquerda sempre que encontramos trombos, ou que o paciente tenha apresentado problemas embólicos na sua evolução pré-operatória.

Em nossa série, os dados concordam com os encontrados na literatura, já que o número de reoperaçōes foi maior naqueles que tinham fibrilação atrial prévia. Assim, $43(59,7 \%)$ dos 72 portadores de ritmo sinusal necessitaram de outra cirurgia, contra $21(75 \%)$ dos 28 que apresentavam fibrilação atrial.

Não podemos deixar de lembrar da função ventricular, que, se não atua na valva propriamente dita, pode 
ARNONI, A. S.; COUTO, W. J.; DINKHUYSEN, J. J.; CHACCUR, P.; MENEGHELO, Z. M.; SOUZA, L. C. B.; JATENE, A. D.; PAULISTA P. P. - Comissurotomia mitral: como evoluem os pacientes a longo prazo? Rev. Bras. Cir. Cardiovasc., 5(2): 106-112, 1990.

prejudicar, e muito, a evoluçāo. KENNEDY et alii ${ }^{20}$ acham que o tratamento cirúrgico não tem grande efeito na função ventricular, pelo menos em período curto de seguimento.

\section{CONCLUSÕES}

Os bons resultados nas comissurotomias mitrais dependem de uma série de fatores, entre os quais destacamos a qualidade da valva, o ritmo cardíaco, a idade do paciente e o sexo. A recorrência da febre reumática também deve ser considerada. Mas a perfeita correão da estenose, sem deixar gradiente residual importante e não produzindo insuficiência, parece ser o mais importante para a boa evolução a longo prazo.

Os nossos pacientes, com evolução média de 19,8 anos, dos quais 52 ainda se encontram com sua valva natural, demonstram bem que a comissurotomia mitral pode ser realizada com excelentes resultados a longo prazo.

RBCCV 44205-113

\begin{abstract}
ARNONI, A. S.; COUTO, W. J.; DINKHUYSEN, J. J.; CHACCUR, P.; MENEGHELO, Z. M.; SOUZA, L.
C. B.; JATENE, A. D.; PAULISTA, P. P. - Open mitral commissurotomy: how are the longterm results?

Rev. Bras. Cir. Cardiovasc., 5(2): 106-112, 1990.

ABSTRACT: To evaluate a longterm evolution of patients who were submitted to an open mitral commissurotomy, we studied 100 patients who were operated upon between 1962 and 1975 . They have had follow-up in the Instituto Dante Pazzanese de Cardiologia for the minimum of 14 years. Sixty-six were women and 24 were men. Their ages ranged from 11 to 50 years, and the media was 30.8 years. Sixty-five patients were reoperated and 18 of them were submitted to a new commissurotomy; in one a revascularization was done; in one the replacement of tricuspide valve; and in 45 times the valve was replaced ( 43 biological prosthesis and 2 metalics). The medium time among he first and the second surgery was 13.6 years. There wasn't any death among the reoperations. Thirty five patients keep in evolution of the first surgery, the medium time is 17.2 years (minimun 14 years and maximum 27 years). Ten are in the NYHA functional class I, 17 in II, seven in III and one in class IV. After this time of evolution, 52 patients are with their natural valves, showing good evolution of the mitral commissurotomy.
\end{abstract}

DESCRIPTORS: mitral commissurotomy, late follow-up, late follow-up; heart valves, mitral, surgery.

\section{REFERÊNCIAS BIBLIOGRÁFICAS}

1 AKINS, C. W.; KIRKLIN, J. K.; BLOCK, P. C.; BUCKLEY, M. J.; AUSTEN, G. - Preoperative evaluation of subvalvar fibrosis in mitral stenosis. Circulation, 60: 71-76, 1979.

2 ANTUNES, M. J.; MAGALHÃES, M. P.; COLSEN, P. R.; KINSLEY, R. H. - Valvoplasty for rheymatic mitral valve disease: a surgical challenge. J. Thorac. Cardiovasc. Surg., 94: 44-56, 1987.

3 ARNONI, A. S.; SALERNO, P. R.; HENRIQUES NETO, A. T. M.; DINKHUYSEN, J. J.; CHACCUR, P.; ABDULMASSIH NETO, C.; NAVARRO, S. L.; ESTEVES, C. A.; RAMOS, A. I. O.; SOUSA, J. E. M. R.; JATENE, A. D.; SOUZA, L. C. B.; PAULISTA, P. P. - O papel do cirurgiāo nas valvopatias reumáticas tratadas com valvoplastia percutânea. Rev. Bras. Cardiovasc., 4: 51-63, 1989.

4 BAILEY, C. P. - The surgical treatment of mitral stenosis (mitral comissurotomy). Dis. Chest., 15: 377-389, 1949.
5 CAMARANO, G. P.; POMERANTZEFF, P. M. A.; JATENE, F.; BARBERO-MARCIAL, M.; MORAES, A. V.; GRINBERG, M.; BELLOTTI, G.; PILEGGI, F. J. C.; JATENE, A. D. - Avaliação do tratamento cirúrgico da valva mitral através de ecocardiografia intra-operatória. Arq. Bras. Cardiol., 50: 237-242, 1988.

6 CARPENTIER, A.; CHAUVAUD, S.; FABIANI, J. N.; DELOCHE, A.; RELLAND, J.; LESSANA, A.; D'ALLAINES, C. L.; BLONDEAU, P.; PIWNICA, A.; DUBOST, C. Reconstructive surgery of mitral valve incompetence: ten year appraisal. J. Thorac. Cardiovasc. Surg., 79: 338-348, 1980.

7 COSGROVE, D. M.; CHAVES, A. M.; LYTLE, B. W.; GILL, C. C.; STEWART, R. W.; TAYLOR, P. C.; GOORMASTIC, M.; BORSH, J. A.; LOOP, F. D. - Results of mitral valve reconstruction. Circulation, 74: 182-187, 1986.

8 CUTLER, E. C. \& BECK, C. S. - The present status of the surgical procedure in chronic valvular disease of the heart. Arch. Surg., 18: 403-416, 1929. 
ARNONI, A. S.; COUTO, W. J.; DINKHUYSEN, J. J.; CHACCUR, P.; MENEGHELO, Z. M.; SOUZA, L. C. B.; JATENE, A. D.; PAULISTA P. P. - Comissurotomia mitral: como evoluem os pacientes a longo prazo? Rev. Bras. Cir. CardiovasC., 5(2): 106-112, 1990.

9 DEKKER, A.; BLACK, H.; VON LICHTENBERG, F. - Mitral valve restenosis: a pathologic study. J. Thorac. Cardiovasc. Surg., 55: 436-446, 1968.

10 DURAN, C. G.; REVUELTA, J. M.; GAITE, L.; ALONSO, C.; FREITAS, M. G. - Stability of mitral reconstructive surgery at 10-12 years for predominantly rheumatic valvular disease. Circulation, 78: 191-196, 1988.

11 GALlOWAY, A. C.; COLVIN, S. B.; BAUMANN, G.; ESPOSITO, R.; VOHRA, R.; HARTY, S.; FREEDBERG, R.; KRONZON, I.; SPENCER, F. C. - Long-term results of mitral valve reconstruction with Carpentier techniques in 148 patients with mitral insufficiency. Circulation, 78 (Supl. 1): 97-105, 1988.

12 GERMANI, S.; MESSMER, B. J.; HALLMAN, G. C. - Open mitral commissurotomy. J. Thorac. Cardiovasc. Surg., 62: 366-368, 1971.

13 GROOS, R. I.; CUNNINGHAM Jr., J. N.; SNIVELY, S. L.; CATINELLA, F. P.; NATHAM, I. M.; ADAMS, P. X.; SPENCER, F. C. - Long-term results of open mitral commissurotomy: ten-year follow-up study of 202 patients. Am. J. Cardiol., 47: 821-825, 1981.

14 HALSETH, W. L.; ELLIOT, D. P.; WALKER, E. L.; SMITH, E. A. - Open mitral commissurotomy: a modern re-evaluation. J. Thorac. Cardiovasc. Surg., 96: 33-38, 1988.

15 HARKEN, D. E.; ELLIS, L. B.; WARE, P. F.; NORMAN, L. R. - The surgical treatment of mitral stenosis: I. Valvuloplasty. N. Engl. J. Med., 239: 804-808, 1948.

16 HERMANN, H. C.; WILKINS, G. T.; ABASCAL, V. M.; WEYMAN, A. E.; BLOCK, P.C.; PALACIOS, I. F. - Percutaneous balloon mitral valvotomy for patients with mitral stenosis. J. Thorac. Cardiovasc. Surg., 96: 33-38, 1988

17 HIGGS, L. M.; GLANCY, D. L.; O'BRIEN, K. P.; EPSTEIN, S. E.; MORROW, A. G. - Mitral restenosis: an uncommon cause of recurrent symptoms following mitral commissurotomy. Am J. Cardiol, 26: 34-37, 1970.

18 HOUSMAN, L. B.; BONCHEK, L.; LAMBERT, L.; GRUNKEMEIER, G.; STARR, A. - Prognosis of patients after open mitral commissurutomy: actuarial analysis of late results in 100 patients. J. Thorac. Cardiovasc. Surg., 73: 742-745, 1977.

19 INOUE, K.; OWAGI, T.; NAKAMURA, T.; KITAMURA, F.; MYIAMOTO, N. - Clinical application of transvenous mitral commissurotomy by a new balloon catheter. J. Thorac. Cardiovasc. Surg., 87: 394-402, 1984.

20 KENNEDY, J. W.; DOCES, J. G.; STEWART, D. K. Left ventricular function before and following surgical treatment of mitral valve disease. Am. Heart J., 97: 592-598, 1979

21 KYO, S.; TAKAMOTO, S.; MATSUMURA, M.; ASANO, H.; YOKOTE, Y.; MOTOYAMA, T.; OMOTO, R. - Immediate and early post-operative evaluation of results of cardiac surgery by transesophageal two dimensional doppler echocardiography. Circulation, 76 (Supl. 5): 113-121, 1987.
McGOON, D. C. - Repair of mitral insufficiency due to ruptured chordae tendinae. J. Thorac. Cardiovasc. Surg., 39: 357-362, 1960.

23 MANTEUFFEL-SZOEGE, L.; NOWICKY, J.; WASNIEWSKA, M.; SITKOWSKI, W.; TURSKI, C. - Mitral commissurotomy: results in 1700 cases. J. Cardiovasc. Surg., 11: 350-354, 1970.

24 MULLIN, M. J.; ENGELMAN, R. M.; ISOM, O. W.; BOYD, A. D.; GLASMANN, E.; SPENCER, F. C. - Experience with open mitral commissurotomy in 100 consecutive patients. Surgery, 76: 974-982, 1974.

25 PALACIOS, I.; BLOCK, P. C.; BRANDI, S.; BLANCO, P.; CASAL, H.; PULIDO, J. I.; MUNOZ, S.; D'EMPAIRE, G.; ORTEGA, M. A.; JACOBS, M.; VLAHAKES, G. Percutaneous balloon valvotomy for patiens with severe mitral stenosis. Circulation, 75: 778-784, 1987.

26 SMITH, W. M.; NEUTZE, J. M.; BARRATT-BOYES, B. G.; LOWE, J. B. - Open mitral valvotomy: effect of preoperative factors on results. J. Thorac. Cardiovasc. Surg., 82: 738-751, 1981.

27 STARR, A. \& EDWARDS, M. L. - Mitral replacement: clinical experience with a ball valve prosthesis. Ann. Surg., 154: 726, 1961.

28 VEGA, J. L.; FREITAS, M.; MARTINEZ, R.; GALLO, J. I.; GUTIERREZ, J. A.; COLMAN, T.; DURAN, M. G. -Open mitral commissurutomy. Ann. Thorac. Surg., 31: $266-270,1981$

\section{Discussão}

\section{DR. WANDERLEY NETTO \\ Maceió, $A L$}

O trabalho do Dr. Arnoni procura nos trazer uma luz a respeito da evolução a longo prazo dos pacientes submetidos a valvotomia mitral aberta. Por dificuldades já expostas pelo autor, não foi possível o seguimento dos pacientes consecutivamente operados e por isso não temos informaçōes com análise atuarial predictiva da efetividade de operação, sobrevida, etc. Apesar dessa limitação, o trabalho traz informaçōes importantes: 1) o cirurgião pode fazer uma 2 : comissurotomia, com bom resultado tardio. 2) os pacientes mais jovens e os operados em grau funcional avançado (mais tempo de sintomas) e alteração do ritmo são mais reoperados e têm resultado funcional e sobrevida piores. Essas informações indicam que, uma vez diagnosticada uma estenose mitral grave, devemos recomendar a cirurgia, independentemente da gravidade dos sintomas e que, nos pacientes jovens, devemos fazer profilaxia secundária rigorosa de cardite reumática, para que possamos melhorar os resultados tardios, nesse grupo. Acreditamos que a efetividade da valvotomia dependerá, basicamente (assegurando-se uma operação bem realizada), do estado da valva, das alteraçōes secundárias provocadas pela 
ARNONI, A. S.; COUTO, W. J.; DINKHUYSEN, J. J.; CHACCUR, P.; MENEGHELO, Z. M.; SOUZA, L. C. B.; JATENE, A. D.; PAULISTA P. P. - Comissurotomia mitral: como evoluem os pacientes a longo prazo? Rev. Bras. Cir. Cardiovasc., 5(2): 106-112, 1990.

duração da doença e da evoluçāo da doença reumática, que é crônica, auto-imune e tem comportamento diferente em cada paciente, e até regiōes. A título de ilustração, no nosso Serviço, operamos, até o mês de março último, 842 pacientes para tratamento de valvopatias. Entre julho de 1978 e dezembro de 1982, fizemos um levantamento dos pacientes que se submeteram a valvotomia mitral e observamos a sobrevida e a necessidade de uma nova operaçāo, que foi, em nove anos, de $94 \%$ e $68 \%$, respectivamente. Quando comparamos as reoperações por valvotomia e troca de valva, observamos que o índice de reoperação foi muito próximo, indicando que, na doença reumática, qualquer que seja a operaçāo, existe sempre a possibilidade de uma nova operação. Finalizando, gostariamos de cumprimentar o Dr. Arnoni pelo seu trabalho, pelo tema abordado, sempre oportuno em nosso país e formularíamos três questōes: 1) quais os achados cirúrgicos predominantes nas reoperaçōes? 2) como eram, na 1: operação, os doentes que estão em classe III e IV? 3) os pacientes jovens foram submetidos a profilaxia para a doença reumática, e até que idade? Encerrando, agradecemos à direção do Congresso por este honroso convite, bem como a atenção dos senhores. Muito obrigado.

\section{DR. ISEU AFFONSO DA COSTA Curitiba, $P R$}

Cumprimento o Dr. Arnoni e seus colaboradores, por sua contribuição a um velho tema que, hoje, após 40 anos de história da moderna cirurgia da valva mitral, ganha interesse, entre outros motivos, pelo surgimento da valvoplastia percutânea. Este tema é, para nós, também, motivo de estudo contínuo. No confronto entre o tratamento cirúrgico e as valvoplastias percutâneas, tem importância, também, o risco do procedimento. Notamos que o Dr. Arnoni registra mortalidade de 1,1\% para a ccmissurotomia aberta, no I.D.P.C., o que está de acordo com a literatura, que mostra, inclusive, menor mortalidade do que para a comissurotomia fechada. Revimos, recentemente, com o mesmo objetivo que o Dr. Arnoni, os resultados imediatos da comissurotomia aberta, durante a década de 1979 a 1988, no Hospital de Clínicas da Universidade Federal do Paraná. Os resultados imediatos são muito satisfatórios e, naturalmente, o próximo passo é realizar uma análise tardia, tal como fizeram o Dr. Arnoni e seus colaboradores. Estudando o presente trabalho, restaram algumas dúvidas, que o Dr. Arnoni, certamente, poderá esclarecer. Em primeiro lugar, o estudo refere-se a amostra de 100 casos, de um universo de $1841(5,4 \%)$. Metodologicamente, seria necessário comprovar que a amostra não difere, em suas características, da população geral. Certamente, objeções podem ser levantadas de que a amostra apresente viezes importantes. Um dado que julgo importante é o de que o estudo, selecionando 100 pacientes que tiveram seguimento regular no I.D.P.C., exclui, ipso facto, pacientes que faleceram tardiamente. $\mathrm{Na}$ verdade, foi analisada a evolução de 100 sobreviventes tardios à comissurotomia mitral. Por outro lado, há referência ao estado funcional dos pacientes no pós-operatório, mas năo no pré-operatório. Sabemos que o resultado tardio da comissurotomia é influenciado por este fator, e algumas conclusōes poderiam ser tiradas a partir de sua análise. Dos 100 pacientes, 65 foram a reoperação (aos quais devemos acrescentar mais quatro, com três operaçōes e dois, com quatro operaçōes). Das 65 reoperaçōes (63 foram na valva mitral), portanto, apenas 37 foram operações que ainda estavam satisfatórias no período estudado. Entretanto, dos 35 pacientes que não necessitaram nova intervenção, oito estão nas classes III e IV NYHA, de modo que temos 27 pacientes que podem ser considerados satisfatórios (27\%). Outro fato que nos chamou a atenão foi a distribuição etária nos resultados. Assim, de oito pacientes operados com idades abaixo de 20 anos, seis foram reoperados. Caberá concluir que a valvoplastia percutânea é a opção inicial preferivel neste grupo? Verificamos que, abaixo de 40 e, principalmente, abaixo de 30 anos de idade, a probabilidade de reoperação é de mais de $70 \%$, o que seria utilizável, como argumento para opção inicial pela valvoplastia. Para esclarecer essas dúvidas, necessitamos de outros estudos, como o do Dr. Arnoni e colaboradores, bem como de semeIhante análise dos resultados de valvoplastia percutânea, antes de ser possivel uma decisão bem fundamentada sobre o problema.

\section{DR. ARNONI \\ (Encerrando)}

Gostaria de agradecer os comentários do Dr. Wan. derley e do Dr. Iseu, e salientar a dificuldade que existe, em nosso país, de se conseguir a evolução a longo prazo dos pacientes. Por esse motivo, nos preocupamos em analisar os pacientes que fizeram sempre sua evolução em nossa Instituição, procurando saber a sobrevida do procedimento e não dos pacientes. Como todo trabalho retrospectivo, tivemos dificuldades em saber como eram as valvas no primeiro procedimento, o que nos impediu, inclusive, de comparar nossos resultados com os da literatura, no que diz respeito às calcificaçōes e comprometimento subvalvar, que, seguramente, devem ter interferido na evolução tardia. Quanto à profilaxia, ela é realizada sempre com emprego de penicilina benzatina de três em três semanas. Com relação aos problemas levantados pelo $\mathrm{Dr}$. Iseu, quero crer que a valvoplastia percutânea possa ser um bom procedimento em pacientes selecionados. Aqueles que têm comprometimento subvalvar, que correspondem a 60 ou $70 \%$ dos casos, não devem ter resultados bons a médio prazo, uma vez que o balão só abre as comissuras, mas não consegue atuar no subvalvar. Além disso, esse é um procedimento novo, que vai precisar de uma análise a longo prazo, para poder compará-lo com um procedimento tão bem estabelecido e com resultados bons a longo prazo, como a comissurutomia mitral. 